\title{
Prise en charge des brûlures dans un service des urgences de France : évaluation des pratiques professionnelles
}

\section{Management of Burns in a French Emergency Department: Evaluation of Professional Practices}

\section{Czolnowski · E. Duret · D. Baugnon · M.-R. Losser}

Reçu le 5 janvier 2018 ; accepté le 13 juillet 2018

(C) SFMU et Lavoisier SAS 2018

Résumé Objectif : Décrire l'épidémiologie des brûlures, le parcours de soins dans un service des urgences et l'adéquation du traitement avec celle des recommandations de la Société française de brûlologie.

Matériel et méthode : Étude observationnelle prospective monocentrique sur un an incluant toute brûlure cutanée de deuxième et troisième degrés prise en charge aux urgences de Verdun (France) avec une analyse rétrospective téléphonique à trois mois.

Résultats : Sur un an, 117 patients ont été inclus, âgés de 27 \pm 22 ans, avec une brûlure d'origine thermique (92\%) surtout par liquide chaud (41\%). Les lésions étaient essentiellement inférieures à $5 \%$ de la surface cutanée $(87 \%$ des patients) surtout de deuxième degré superficiel $(80 \%$ des lésions). Un protocole de pansement et de remplissage vasculaire non recommandé était majoritairement prescrit. Neuf patients furent transférés le jour même vers un centre de référence, cinq l'ont été à 48 heures. L'usage de la télémédecine a échoué par dysfonctionnement du logiciel. Le suivi était essentiellement réalisé par un médecin urgentiste sur un court terme. Sur les 71 dossiers réévalués téléphoniquement à trois mois, seuls quatre cas ont révélé un retentissement personnel ou professionnel.

Conclusion : L'établissement d'un réseau de soins articulé entre le service des urgences, la médecine de ville et les centres de traitement des brûlés semble démontrer un intérêt tant

D. Czolnowski $(\bowtie) \cdot$ E. Duret $\cdot$ D. Baugnon

Samu 55, centre hospitalier de Verdun,

2, rue d'Anthouard, F-55100 Verdun, France

e-mail : dczolnowski@ch-verdun.fr

\section{M.-R. Losser}

Service d'anesthésie-réanimation, CHRU de Nancy,

5, rue du Morvan, F-54500 Vandœuvre-lès-Nancy, France

Centre des brûlés, CHR de Metz-Thionville,

1, rue du Friscaty, F-57100 Thionville, France

Faculté de médecine, université de Lorraine,

5, rue du Morvan, F-54500 Vandœuvre-lès-Nancy, France sur le plan de la qualité des soins initiés aux urgences que sur le suivi et la prévention des complications aiguës et tardives de la brûlure.

Mots clés Brûlures thermiques · Épidémiologie · Télémédecine $\cdot$ Réseaux de soins $\cdot$ Pansements

Abstract Objective: Description of epidemiology of burns, care pathways in emergency department and adequacy of treatment in relation to the guidelines of the French Society of Burns.

Material and method: Monocentric prospective observational study over a year, including all second- and third-degree burns treated at the emergency department of Verdun (France), with a 3-month retrospective analysis over the phone.

Results: In 1 year, 117 patients were enrolled, aged $27 \pm 22$, with a thermal burn $(92 \%)$ predominantly by hot liquid (41\%). The lesions were essentially less than $5 \%$ of the total body surface ( $87 \%$ of the patients), especially of second superficial degree ( $80 \%$ of the injuries). A non-recommended dressing protocol and vascular filling were mainly prescribed. Nine patients were transferred to a referral centre on the same day, five of them were sent within 48 hours. The use of telemedicine failed due to a software malfunction. The follow-up was essentially carried out by an emergency physician on short-term basis. Out of the 71 records re-evaluated over the phone at 3 months, only four cases revealed personal or professional repercussions.

Conclusion: The establishment of a care network in connection with emergency department, general practice and reference centres seems to benefit the patients both in terms of quality of care initiated at the emergency department and follow-up and prevention of early and late complication of burn.

Keywords Thermal Burns · Epidemiology $\cdot$ Telemedicine $\cdot$ Care network $\cdot$ Dressing 


\section{Introduction}

Les lésions par brûlures, d'origine accidentelle ou intentionnelle, sont responsables de séquelles physiques, psychologiques avec un certain retentissement socioprofessionnel aussi bien pour la victime que son entourage. En 2011, l'Institut de veille sanitaire dénombrait environ 12000 hospitalisations pour brûlure en France métropolitaine dont $43 \%$ dans les 24 centres de référence [1]. Pour les médecins urgentistes, la prise en charge en ambulatoire d'une brûlure exige une connaissance en matière de physiopathologie, de thérapeutiques, de prévention ainsi qu'une connaissance des réseaux de soins spécialisés [2]. Nombreux sont les hôpitaux et trauma centers ne disposant pas de service spécialisé en brûlure. Depuis 2014, le schéma interrégional d'organisation des soins de la zone de défense Est (SIOS 2) a été réactualisé et a présenté une procédure opérationnelle standardisée interrégionale pour les brûlés graves (POS Grands Brûlés), validée par la Société française d'étude et de traitement de la brûlure, devenue en 2016 la Société française de brûlologie (SFB). Il définit aussi des territoires médicaux afin d'orienter les patients vers des centres de traitement des brûlés (CTB) [3]. Les centres demandeurs doivent être en mesure de contacter facilement les centres de référence ; pour cela, ils bénéficient, au travers d'une application opérationnelle nommée « Odys Grands Brûlés » $\left(\right.$ Covotem ${ }^{\mathrm{TM}}$ et IDO-in ${ }^{\circledR}$, Dijon, France) [4], d'échanges sécurisés d'iconographies et d'une expertise avec le concours de la régulation médicale du Cen- tre 15 garantissant une qualité de soins lors de la prise en charge d'une victime en extra- ou intrahospitalier [5]. À ce jour, rares sont les études préhospitalières en France portant sur l'analyse des circonstances des brûlures, leurs caractéristiques, la prise en charge en ambulatoire et leur évolution dans le temps. Nous avons cherché à décrire l'épidémiologie des brûlures, le parcours de soins dans un service des urgences et l'adéquation du traitement avec les recommandations validées par la SFB.

\section{Matériel et méthode}

Il s'agissait d'une étude observationnelle prospective monocentrique, incluant les patients consultant au service des urgences du centre hospitalier de Verdun et dont le motif d'admission était une brûlure cutanée, couplée avec une analyse rétrospective à trois mois dans le cadre d'une évaluation des pratiques professionnelles. La période d'inclusion allait du $1^{\text {er }}$ mai 2015 au 31 avril 2016. Le critère d'inclusion était la survenue d'une brûlure cutanée de deuxième ou troisième degré chez des patients adultes et pédiatriques. Le critère d'exclusion était la présence d'une brûlure exclusivement de premier degré. Plusieurs critères de la POS Grands Brûlés concernant la gestion des brûlures ont été utilisés afin d'évaluer les bonnes pratiques du service des urgences de Verdun (Tableaux 1,2). Ces critères, validés par la SFB, ont été pris en compte comme actuelle référence [6-8]. Un formulaire

Tableau 1 Critères d'hospitalisation d'un brûlé vers un centre de grands brûlés, issus de la procédure organisationnelle standardisée du SIOS 2 (janvier 2014)

\begin{tabular}{|c|c|c|c|}
\hline & \multicolumn{3}{|c|}{ Décision d'hospitalisation } \\
\hline & Prise en charge ambulatoire & Hospitalisation & $\begin{array}{l}\text { Hospitalisation en centre } \\
\text { spécialisé }\end{array}$ \\
\hline SCB & $<10 \%$ & $10 \%<\mathrm{SCB}<20 \%$ & $\geq 20 \%$ \\
\hline Surface brûlée au $3^{\mathrm{e}}$ degré & $<3 \%$ & $3 \%<3^{\mathrm{e}}$ degré $<5 \%$ & $>5 \%$ \\
\hline $\begin{array}{l}\text { Localisations particulières : } \\
\text { face, mains, pieds, périnée }\end{array}$ & $\begin{array}{l}<3 \% \text { et absence de lésion } \\
\text { au troisième degré, de lésions } \\
\text { de la tête, des mains, des pieds } \\
\text { ou du périnée }\end{array}$ & $<3 \%$ au $3^{\mathrm{e}}$ degré & $\geq 3 \%$ au $3^{\mathrm{e}}$ degré \\
\hline Brûlures électriques & & $\begin{array}{l}\text { Brûlures électriques faible } \\
\text { voltage sans lésion } \\
\text { cardiologique ni brûlure } \\
\text { profonde }\end{array}$ & $\begin{array}{l}\text { Brûlures de haut voltage } \\
\text { avec brûlures profondes et/ } \\
\text { ou atteinte viscérale }\end{array}$ \\
\hline Lésions d'inhalation de fumées & Aucune & $\begin{array}{l}\text { Si } \mathrm{SCB}<10 \% \text { et absence } \\
\text { de } 3^{\mathrm{e}} \text { degré de la face }\end{array}$ & $\begin{array}{l}\text { Si SCB }>10 \% \text { ou si } 3^{\mathrm{e}} \text { degré } \\
\text { de la face }\end{array}$ \\
\hline \multicolumn{4}{|c|}{ Élargir les indications d'hospitalisation si problèmes sociaux } \\
\hline Les enfants de moins de 5 ans & et $\mathrm{SCB}>5 \%$ et $/$ ou brûlures prof & ondes seront hospitalisés & \\
\hline
\end{tabular}


Tableau 2 Synthèse des recommandations de la prise en charge d'un brûlé à la phase aiguë

\begin{tabular}{|c|c|c|}
\hline Soins & Qui & Quels moyens \\
\hline Oxygénation & $\begin{array}{l}\text { Tous les brûlés graves } \\
\text { Les victimes d'incendie }\end{array}$ & $\begin{array}{l}\text { Masque } \mathrm{O}_{2} \\
\text { Masque haute concentration }\end{array}$ \\
\hline Intubation & $\begin{array}{l}\text { Trouble de la conscience }(\text { Glasgow }<8 \text { ) } \\
\text { Détresses respiratoires } \\
\text { Brûlures majeures de la tête et du cou } \\
\text { Modification de la voix dans un contexte } \\
\text { d'incendie } \\
\text { Brûlures }>50 \% \text { surface cutanée } \\
\text { et transport long }\end{array}$ & $\begin{array}{l}\text { Préoxygénation puis crash induction (étomidate }+ \\
\text { succinylcholine) }\end{array}$ \\
\hline Ablation des vêtements & Brûlure par liquides chauds & Retrait des vêtements non adhérents \\
\hline Refroidissement & Seulement la brûlure & Eau froide (non glacée), température entre 10 et $20^{\circ}$ \\
\hline de la brûlure & $\begin{array}{l}\text { Contre-indiqué chez le patient en choc, } \\
\text { trouble de la conscience et/ou brûlure }> \\
10 \% \text { surface corporelle }\end{array}$ & $\begin{array}{l}\text { Hydrogels antibrûlures } \\
\text { Brûlures dans les } 15 \text { premières minutes } \\
\text { Inutile au-delà de } 30 \text { minutes }\end{array}$ \\
\hline Réchauffement & Toujours & $\begin{array}{l}\text { Couverture isotherme } \\
\text { Réchauffement du vecteur }\end{array}$ \\
\hline Perfusion & $\begin{array}{l}\text { Troubles de la conscience } \\
\text { Surface cutanée brûlée }>10 \%\end{array}$ & $\begin{array}{l}\text { Périphérique peau saine } \\
\text { Sinon périphérique peau brûlée } \\
\text { Centrale peau saine sinon centrale peau brûlée sinon } \\
\text { intraosseuse } \\
\text { Remplissage vasculaire }\end{array}$ \\
\hline Analgésie & Tous les brûlés & $\begin{array}{l}\text { Palier } 1 \text { : paracétamol } \\
\text { Palier } 3: \text { morphine intraveineuse en titration } \\
\text { Mélange équimolaire de protoxyde d'azote et d'oxygène }\end{array}$ \\
\hline Anesthésie & Intubation et gestes douloureux & $\begin{array}{l}\text { Kétamine } \\
\text { Midazolam } \\
\text { Gamma-hydroxybutyrate de sodium }\end{array}$ \\
\hline Sonde urinaire & $\begin{array}{l}\text { Brûlures des organes génitaux externes } \\
\text { Surveillance remplissage }\end{array}$ & Intérêt des sondes à thermistance \\
\hline Soins locaux & $\begin{array}{l}\text { Toute brûlure }>2^{\mathrm{e}} \text { degré } \\
\text { Après refroidissement }\end{array}$ & $\begin{array}{l}\text { Lavage avec savon ou chlorhexidine diluée } \\
\text { Rasage des zones brûlées } \\
\text { Mise à plat des phlyctènes } \\
\text { Retrait des lambeaux cutanés Rinçage sérum physiologique } \\
\text { Couche épaisse d'acide hyaluronique couplé à la sulfadiazine } \\
\text { argentique } \\
\text { Panser avec compresses stériles } \\
\text { Fermer avec une bande ou un filet tubulaire sans serrer }\end{array}$ \\
\hline
\end{tabular}

d'information écrit et exhaustif concernant cette étude était disponible pour chaque patient à l'accueil du service des urgences. L'accès et le traitement des données informatisées du patient ont été validés auprès de la Commission nationale de l'informatique et des libertés (numéro d'enregistrement : 1959766). Cette étude a été validée par le comité d'éthique du centre hospitalier de Verdun. Chaque patient inclus bénéficiait d'un examen clinique complet orienté par un questionnaire mis à disposition des 18 médecins urgentistes du service dans le logiciel ResUrgences ${ }^{\circledR} \quad$ (Berger-Levrault,
Boulogne-Billancourt, France) et sur le cahier d'intervention en services mobiles d'urgence et de réanimation (Smur). Chaque patient inclus a été informé oralement d'une réévaluation téléphonique trois mois après le passage au service des urgences. Deux tentatives d'appel téléphonique au cours de ce mois de réévaluation étaient acceptées. Les dossiers médicaux étaient codés grâce à la Classification internationale des maladies, dixième révision puis transférés informatiquement vers un second tableau où le numéro d'identification des patients avait été converti pour garantir l'anonymat. 


\section{Analyse statistique}

Pour les variables qualitatives et binaires, nous avons calculé des pourcentages. Pour les variables quantitatives, nous avons utilisé des moyennes ainsi que des médianes. L'unité statistique était le patient. Les données ont été traitées sur le logiciel Microsoft ${ }^{\mathbb{R}}$ Office Excel (Microsoft ${ }^{\mathbb{R}}$, Redmond, Washington, États-Unis).

\section{Résultats}

Notre étude a permis d'inclure 117 patients, soit $0,4 \%$ des passages au service des urgences de Verdun à cette période $(n=29$ 403). L'âge moyen est de $27 \pm 22$ ans. Il a été retrouvé deux pics de populations préférentiels : les enfants âgés de moins de cinq ans et les adultes jeunes actifs de moins de 30 ans (Tableau 3). Le sujet le plus jeune était âgé de sept mois, le plus âgé de 83 ans. La fréquence des accidents semblait se majorer discrètement en début de période estivale. Les circonstances des accidents de brûlure étaient essentiellement d'origine domestique (87\%, $n=102$ ). Dans la majorité des cas, la nature de la brûlure était thermique ( $91 \%, n=107)$, incluant les liquides chauds $(41 \%, n=48)$, les surfaces solides chaudes $(37 \%, n=43)$ et les flammes $(13 \%, n=16)$. Les brûlures chimiques par base et acide ne représentaient que $4 \%$ des cas $(n=5)$, toutes, au cours d'accidents du travail. Les lésions décrites, parfois de degrés différents pour un même patient $(n=122)$, étaient pour la plupart de deuxième degré superficiel. L'essentiel des brûlures avait une surface cutanée estimée inférieure à $5 \%$ de la surface corporelle (Tableau 3). Le score de Baux est un indice de pronostic vital d'un brûlé résultant de la somme de la surface cutanée brûlée et de l'âge du patient

Tableau 3 Répartition des patients selon le pourcentage des surfaces cutanées brûlées. Dans cette cohorte préhospitalière, on retrouve les trois pics de population exposés le plus aux brûlures

\begin{tabular}{|lllll|}
\hline $\begin{array}{l}\text { Classe } \\
\text { d'âge } \\
\text { (ans) }\end{array}$ & Total & $<\mathbf{5} \%$ & $\mathbf{5 - 1 0} \%$ & $\mathbf{1 1 - 2 5} \%$ \\
\cline { 2 - 5 } $0-4$ & 27 & 24 & 1 & 2 \\
$5-19$ & 18 & 15 & 3 & 0 \\
$20-39$ & 37 & 35 & 0 & 2 \\
$40-69$ & 31 & 26 & 4 & 1 \\
69 et + & 4 & 2 & 2 & 0 \\
Total & 117 & 102 & 10 & 5 \\
\hline
\end{tabular}

Les résultats sont exprimés en nombre (\%) ; SCB : surface cutanée brûlée qui, lorsqu'il est supérieur à 100, laisse présager un pronostic vital engagé. Dans cette étude, cet indice était en moyenne de $46 \pm 16$ (chez les sujets âgés de plus de 20 ans, $n=72$ ). Il n'y a pas eu de décès recensé lors de la prise en charge initiale. Les brûlures étaient retrouvées fréquemment aux extrémités des membres supérieures (59\%), aux membres inférieurs $(13 \%)$ et sur la face $(12 \%)$. Un refroidissement de la zone atteinte a été décrit dans 56 cas (48\%). Parmi ces patients, 16 entraient dans les critères du refroidissement [9]. On dénombrait 32 cas de refroidissement couplés à l'usage de pansements préimprégnés de gels hydriques $(27 \%)$. Dans le service, un pansement associant une compresse imprégnée d'acide hyaluronique couplée à une crème comprenant à la fois de l'acide hyaluronique et de la sulfadiazine argentique a été majoritairement prescrit $(74 \%$, $n=83)$ par rapport à l'application seule de crème à base d'acide hyaluronique et de sulfadiazine argentique couplée à un pansement gras stérile $(n=1)$ (Fig. 1). Dans environ un quart des dossiers, la prise en charge antalgique n'a pas été précisée. Un traitement par palier 1 suffisait (64\%), avec parfois un recours au palier 2 (12\%). Le mélange équimolaire oxygène-protoxyde d'azote (MEOPA) a été efficace à cinq reprises chez des patients pédiatriques. Dans la prise en charge de brûlés graves, neuf patients, adressés au service des urgences et répondant aux critères de gravité de la SFB (Tableau 1), ont été conditionnés puis transférés très précocement vers un CTB, alors que dix patients ont été orientés à 48 heures vers ce centre de référence pour une consultation spécialisée de contrôle. Les remplissages vasculaires renseignés initiés dans le service $(n=8)$ ne respectaient pas les formules recommandées par la SFB [2,6] (Tableau 4). Dans un cas sur cinq, un avis spécialisé a été pris vers un centre de référence $(20 \%, n=23)$. Environ un avis sur deux était accompagné d'un envoi non sécurisé de photos par téléphone personnel. À trois reprises, il y a eu une tentative d'utilisation de la télémédecine sans succès par défaut de fonctionnement du logiciel Odys. Finalement, 71 dossiers $(61 \%)$ de patients ayant consulté sur l'année aux urgences de Verdun pour motif de brûlure au moins du deuxième degré ont pu être réévalués téléphoniquement à trois mois. Quarante-six patients n'ont pas pu être contactés du fait de l'absence de coordonnées téléphoniques dans le dossier médical $(n=16)$, de l'échec du rappel téléphonique du patient à trois mois $(n=23)$ ou parce qu'il s'agissait de patients encore hospitalisés dans un CTB $(n=7)$.

Parmi les patients réévalués téléphoniquement $(n=71)$ et les patients non contactés, mais dont une seconde consultation avait pu être trouvée via le logiciel ResUrgences ${ }^{\circledR}$ $(n=39), 40$ ont bénéficié d'une consultation à 48 heures aux urgences de Verdun pour un contrôle du pansement (36\%) avec une médiane à deux consultations ; un suivi par le médecin traitant ou le pédiatre a été recensé chez 13 patients $(12 \%)$; un suivi dans un CTB a été retrouvé à 
1.

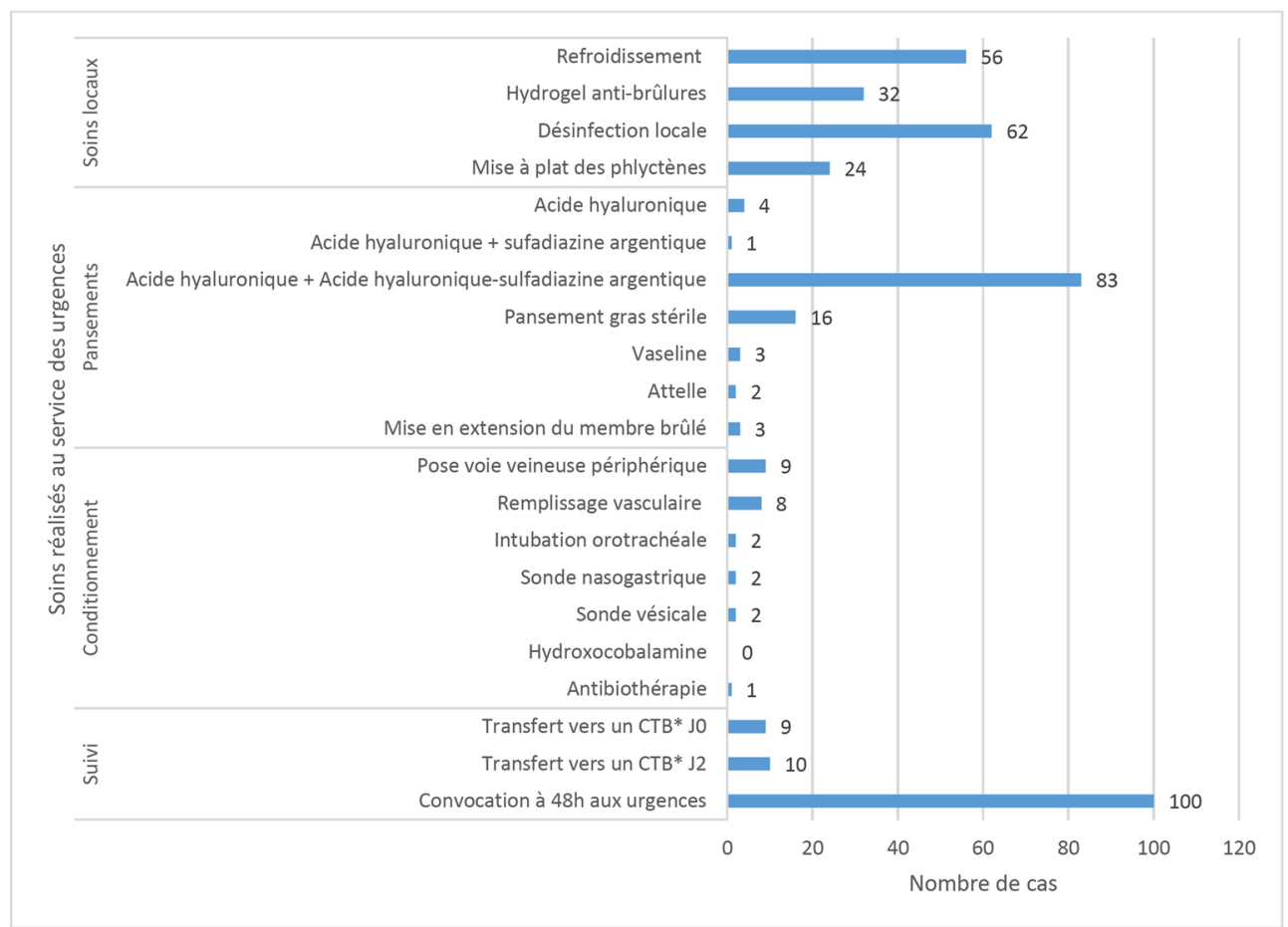

Fig. 1 Prise en charge initiale au service des urgences de Verdun $(n=117){ }^{*}$ CTB : centre de traitement de la brûlure À peine $50 \%$ des patients ont réalisé un refroidissement à l'eau recommandé par la SFB

Tableau 4 Formules de remplissage vasculaire de l'adulte et de l'enfant brûlé

\begin{tabular}{|c|c|c|}
\hline & Formule [2] & 0 à 8 heures \\
\hline $\begin{array}{l}\text { Adulte } \\
\text { et pédiatrie }>\end{array}$ & Evans & $\begin{array}{l}0,5 \mathrm{ml} / \mathrm{kg}^{-1} \text { par } \% \mathrm{SCB}^{-1} \text { avec sérum salé, } 0,5 \mathrm{ml} / \mathrm{kg}^{-1} \text { par } \% \mathrm{SCB}^{-1} \text { avec collö̈des. Besoins } \\
\text { de base adulte : } 2000 \mathrm{ml} \text { de sérum glucosé } 5 \%\end{array}$ \\
\hline $20 \mathrm{~kg}$ & Parkland & $\begin{array}{l}2 \mathrm{ml} / \mathrm{kg} \text { par pourcentage de SCB solution cristalloïde isotonique (avec lactate } \\
\text { ou avec acétate et malate) }\end{array}$ \\
\hline & Percy & $\begin{array}{l}2 \mathrm{ml} / \mathrm{kg} \text { par pourcentage de SCB solution cristalloïde isotonique (avec lactate } \\
\text { ou avec acétate et malate). On soustrait } 20 \mathrm{ml} / \mathrm{kg} \text { à passer sur la première heure }\end{array}$ \\
\hline Pédiatrie $\leq 20 \mathrm{~kg}$ & Carjaval & $\begin{array}{l}\text { Calculer la surface corporelle de l'enfant. Les objectifs des premières } 24 \text { heures sont } \\
\text { un remplissage à } 5000 \mathrm{ml} / \mathrm{m}^{2} \text { de SCB }+2000 \mathrm{ml} / \mathrm{m}^{2} \text { de surface corporelle totale. La moitié } \\
\text { administrée sur les huit premières heures. Remplissage avec une solution } \\
\text { cristalloïde isotonique (avec lactate ou avec acétate et malate) }\end{array}$ \\
\hline
\end{tabular}

48 heures chez dix patients $(9 \%)$ et pour dix patients après une seconde évaluation au service des urgences de Verdun ( $9 \%)$; un suivi par un médecin rééducateur a été proposé pour un enfant. Sur le centre hospitalier de Verdun, on remarquait, pour des raisons non clairement spécifiées dans les dossiers, quatre consultations pour des brûlures ophtalmologiques et quatre consultations auprès des chirurgiens orthopédiques. Pour trois patients réévalués téléphoniquement (4\%), la brûlure a eu un retentissement personnel, et un patient a eu des répercussions sur le plan professionnel. Des douleurs résiduelles à trois mois ont été retrouvées chez trois patients réévalués $(4 \%)$. La prise en charge au service des urgences de la douleur a été jugée efficace dans $99 \%$ des cas. 


\section{Discussion}

Cette étude objective des résultats épidémiologiques au service des urgences de Verdun superposables aux données nationales de 2011 de l'Institut de veille sanitaire [1]. À notre connaissance, il s'agit de la première étude française concernant des cas de brûlures prises en charge dans un service des urgences. Comme décrit dans les études épidémiologiques des passages aux urgences taïwanaises [10] et australiennes [11], les enfants de moins de cinq ans ont la plus grande incidence en matière de brûlure ( $23 \%$ ), suivis par la catégorie des jeunes adultes actifs âgés de moins de 30 ans $(21 \%)$. Cela est expliqué, pour les enfants, par le manque de vigilance et la pauvreté de leurs réflexes liés au développement psychomoteur. Elle reste donc une population cible en matière de prévention. Bien que les brûlures par liquides chauds restent prédominantes, la particularité de cette étude est le nombre de brûlures thermiques par contact $(n=43)$. Ce phénomène résulte de l'utilisation fréquente, en Meuse, de chauffage par poêle en fonte et en céramique. Comme attendu, la majorité des lésions décrites étaient de deuxième degré superficiel pour des surfaces cutanées brûlées essentiellement inférieures à $5 \%$. Les sites les plus souvent atteints étaient les membres supérieurs comme retrouvé dans la littérature $[10,11]$. Cette donnée soulève cependant la question de la justesse de l'estimation de la surface brûlée par les médecins urgentistes. Une étude rétrospective du Yorkshire Regional Burns Unit a démontré que les brûlures de surface de moins de $20 \%$ étaient souvent surestimées par les équipes médicales des services d'urgences, tandis que les brûlures de grande surface étaient souvent sous-estimées, avec pour conséquence un traitement, notamment le remplissage vasculaire, inadapté [12]. Ces éléments nous rappellent l'importance d'une formation continue des professionnels de santé, notamment par des experts dans le domaine de l'évaluation d'une brûlure. Un médecin urgentiste, désigné « référent brûlure », pourrait ainsi actualiser dans son service, en collaboration avec les centres de référence, des protocoles médicaux adaptés aux recommandations validées par la SFB. Les moyens dont les urgentistes disposent pour prendre avis auprès de ces centres de référence restent non structurés (23 avis demandés, $n=117)$ et parfois non sécurisés (11 envois de MMS par téléphone personnel, $n=117$ ). L'usage de la télémédecine par le logiciel Odys dans la prise en charge des brûlés au service des urgences de Verdun est réellement insuffisant, avec un taux d'échec de $100 \%$. Les problèmes informatiques du logiciel (mises à jour du parefeu, sécurisation du logiciel) freinent son usage et nécessitent une amélioration pour éviter le recours aux téléphones personnels et pour garantir aux médecins, notamment en Smur, un réel soutien pour l'organisation du parcours de soins du patient. Tout comme le recommande l'American Burn Association dans son protocole [13], le SIOS 2 établit un réel guide des conduites à tenir pour orienter les patients d'un centre hospitalier vers un centre de référence du traitement des brûlures. Il reprend les critères d'hospitalisation des brûlés graves et met en évidence l'importance de l'évaluation clinique précise de la surface d'une brûlure. Ce support est intéressant dans la prise en charge des brûlés graves, mais notre revue de la littérature n'a pas permis de retrouver de guide pour la prise en charge en ambulatoire des brûlures mineures. Sur le plan thérapeutique, moins de la moitié des victimes ont effectué un refroidissement, et seulement $14 \%$ l'ont réalisé correctement. Une étude épidémiologique britannique, portant sur la description d'une population adulte et pédiatrique admise dans un service d'urgence pour motif de brûlure [14], montre qu'un peu moins de $30 \%$ des patients avaient réalisé des premiers soins appropriés, alors qu'en Australie ce taux s'élève à $39 \%$ [15]. Il a été démontré pourtant que l'application de l'eau fraîche sur une brûlure permet de réduire la douleur, de minimiser l'œdème et l'extension de la lésion ainsi que d'améliorer la cicatrisation [16]. Ce constat prouve un manque d'information auprès de la population sur les premiers gestes de secours à apporter à une victime de brûlure. Dans cette cohorte, les enfants ont été orientés précocement vers un centre de référence de traitement des brûlures (33\%) ou au moins en contrôle au service des urgences à 48 heures (54\%) soit du fait de la complexité des brûlures, soit du fait de la caractéristique de cette population jeune souvent isolée géographiquement des centres de soins, soit du fait d'un contexte social défavorable limitant le recours aux soins et laissant craindre la perte de vue de ces patients. L'essentiel du suivi se fait par les médecins urgentistes à la phase aiguë, mais à moyen terme, aucune mesure de surveillance n'est mise en place, et les patients sont perdus de vue. L'établissement d'un réseau de soins articulé entre les urgences, les centres de référence et les médecins généralistes permettrait d'encadrer les patients victimes de brûlures, de répondre à leurs questionnements et de prévenir les complications à court et moyen termes. Pour cela, de la part des urgentistes, un document fondé sur les recommandations avec des consignes de surveillance et des coordonnées des centres de référence pourrait être remis aux patients, à l'attention des médecins généralistes et des pédiatres. Ce document permettrait d'apporter un support pour les professionnels de santé libéraux pour le suivi de la cicatrisation. L'étude menée en Caroline du Nord dans une centaine de services d'urgences concernant essentiellement des jeunes actifs a démontré que $92 \%$ des consultations pour brûlures minimes avaient pu être gérées par les urgentistes seuls avec un suivi ambulatoire grâce à des instructions remises par le médecin urgentiste directement au patient [17]. Enfin, cette étude a permis de mettre en exergue une double utilisation inappropriée de pansements à base d'acide hyaluronique dans le service d'urgence de Verdun $(74 \%, n=83)$ ainsi qu'une méconnaissance du service sur les modalités 
de remplissage, tant sur le plan qualitatif que quantitatif, du patient brûlé [6].

Il s'agissait d'une étude monocentrique dans un service au nombre de passages annuels inférieur à 30 000, expliquant le faible nombre d'inclusions. La réévaluation à trois mois a été fixée de manière arbitraire, et ce, pour des raisons organisationnelles. Une nouvelle évaluation à 12 mois aurait pu permettre de révéler d'autres complications de la cicatrisation, plus tardives, qui auraient possiblement nécessité un avis spécialisé. De même, nous n'avions pas pris le choix de contacter les centres de référence pour l'évaluation des patients toujours hospitalisés à trois mois. La recherche des antécédents psychiatriques n'a pas été réalisée au cours de cette étude, étant pourtant, selon la SFB, un critère important dans la prise en charge des brûlés. Il est en effet important de connaître les circonstances de l'accident pour considérer la possibilité d'une blessure non accidentelle afin de décider si les patients vulnérables sont en mesure de bénéficier ou non d'un suivi ambulatoire à court et moyen termes. Un score d'analgésie (échelle numérique) à l'arrivée et à la sortie du service des urgences n'a pas été mis en place. Nous n'avons étudié qu'un ressenti subjectif à trois mois. Une étude à Milwaukee s'est penchée sur la prise en charge de l'analgésie en préhospitalier et aux urgences. Elle a démontré ainsi la priorité de la mise en place de protocoles, au travers de scores adaptés à l'âge, et de la sensibilisation des équipes paramédicales à l'usage des opioïdes pour améliorer rapidement et efficacement l'analgésie [18]. Concernant les retentissements socioprofessionnels, les résultats restent informatifs et subjectifs. Ils n'ont pas été établis à partir de recueils standardisés de type échelle de qualité de vie ou encore échelle de l'état de stress post-traumatique, jugés trop longs à réaliser par entretien téléphonique.

\section{Conclusion}

L'établissement d'un réseau de soins articulé entre le service des urgences, la médecine de ville et les centres de traitement des brûlés semble démontrer un intérêt tant sur le plan de la qualité des soins initiés aux urgences que sur le suivi et la prévention des complications aiguës et tardives de la brûlure.

Liens d'intérêts : les auteurs déclarent ne pas avoir de liens d'intérêts.

\section{Références}

1. Pasquereau A, Thélot B (2014) Institut de veille sanitaire. Hospitalisations pour brûlures à partir des données du Programme de médicalisation des systèmes d'information, France métropolitaine 2011 et évolution depuis 2008. http://opac.invs.sante.fr/doc num.php?explnum_id=9447 (Dernier accès le 16 octobre 2017)

2. Carsin H, Le Béver H, Bargues L, Stéphanazzi J (2007) Brûlure. In: EMC Médecine d'urgence. Elsevier-Masson SAS, Paris, 25030-D-40

3. Collège lorrain de médecine d'urgence (2014) POS interrégionale Grands Brûlés. Arbre décisionnel. http://www.colmu.net/spip. php?article1088 (Dernier accès le 30 novembre 2016)

4. Collège lorrain de médecine d'urgence (2014) POS interrégional Grands Brûlés. Formulaire Odys Grands Brûlés application smartphone. http://www.colmu.net/IMG/pdf/annexe_7.pdf (Dernier accès le 30 novembre 2016)

5. Benhamou F, Fyad JP, Chassagne JF (2005) Télémédecine : étude de faisabilité dans le domaine des brûlés. Brûlures 6:138-44

6. Collège lorrain de médecine d'urgence (2014) POS interrégional Grands Brûlés. Prise en charge d'un brûlé grave dans les 48 premières heures. http://www.colmu.net/IMG/pdf/annexe_1_-_pec_ d_un_brule_les_48_premieres_heures_06-02-2014_pdf (Dernier accès le 30 novembre 2016)

7. Collège lorrain de médecine d'urgence (2014) POS interrégional Grands Brûlés. Critères d'appel d'un CGB pour avis et/ou hospitalisation http://www.colmu.net/IMG/pdf/annexe_2_-_pos_gb.pdf (Dernier accès le 30 novembre 2016)

8. Echinard C, Latarjet J (2010) Pansement des brûlures suivies en consultation externe ou en ville. In: Echinard C, Latarjet J (eds) Les brûlures. Masson, Paris, pp 215-7

9. Hudspith J, Rayatt S (2004) ABC of burns first aid and treatment of minor burns. BMJ 328:1487-9

10. Shih-Han C, Yi-Chun C, Tzeng-Ji C, Hsu M (2014) Epidemiology of burns in Taiwan: a nationwide report including inpatients and outpatients. Burns 40:1397-405

11. Wasiak J, Spinks A, Ashby K, et al (2009) The epidemiology of burn injuries in an Australian setting, 2000-2006. Burns 35:1124-32

12. Colis N, Smith G, Fenton OM (1999) Accuracy of burn size estimation and subsequent fluid resuscitation prior to arrival at the Yorkshire Regional Burns Unit. A three year retrospective study. Burns 25:345-51

13. American College of Surgeon (2014) Resources for optimal care of the injured patient. Guidelines for trauma centers caring for brun patients. https://www.facs.org/ /media/files/quality $\% 20$ programs/trauma/vrc\%20resources/resources $\% 20$ for $\% 20$ optimal \%20care.ashx\%20(dernier\%20acc\%C3\%A8s\%20le\%2018/08/ 2018)

14. Chipp E, Walton J, Gorman D, Moiemen N (2008) A 1-year study of burn injuries in a British Emergency Department. Burns 34:516-20

15. Rea S, Kuthubutheen J, Fowler B, Wood F (2005) Burn first aid in Western Australia-do healthcare workers have the knowledge? Burns 31:1029-34

16. Venter THJ, Karpelowsky JS (2007) Cooling of the burn wound: the ideal temperature of the coolant. Burns 33:917-92

17. DeKoning E, Hakenewerth A, Platts-Mills T, Tintinalli J (2009) Epidemiology of burn injuries presenting to North Carolina emergency departments in 2006-2007. Burns 35:776-82

18. Kari B, Brooke E, Clare E, et al (2016) Effect of system-wide interventions on the assessment and treatment of pain by emergency medical services providers. Prehosp Emerg Care 20:752-8 